\title{
Cost Effectiveness of Watch and Wait Versus Resection in Rectal Cancer Patients with Complete Clinical Response to Neoadjuvant Chemoradiation
}

\author{
Christina Liu Cui, MAS ${ }^{1}$, William Yu Luo, MAS ${ }^{1}$, Bard Clifford Cosman, MD, MPH ${ }^{2,3}$, Samuel Eisenstein, MD $^{2}$, \\ Daniel Simpson, MD, MAS ${ }^{4}$, Sonia Ramamoorthy, MD $^{2}$, James Murphy, MD, MS ${ }^{4}$, and Nicole Lopez, MD, MAS ${ }^{2}$ \\ ${ }^{1}$ School of Medicine, University of California, San Diego, La Jolla, CA; ${ }^{2}$ Department of Surgery, Division of Colon and \\ Rectal Surgery, University of California, San Diego Health Systems, La Jolla, CA ; ${ }^{3}$ Veterans Affairs San Diego Medical \\ Center, San Diego, CA; ${ }^{4}$ Department of Radiation Medicine and Applied Science, University of California, San Diego, La \\ Jolla, CA
}

\begin{abstract}
Background. Watch and wait (WW) protocols have gained increasing popularity for patients diagnosed with locally advanced rectal cancer and presumed complete clinical response after neoadjuvant chemoradiation. While studies have demonstrated comparable survival and recurrence rates between WW and radical surgery, the decision to undergo surgery has significant effects on patient quality of life. We sought to conduct a cost-effectiveness analysis comparing WW with abdominoperineal resection (APR) and low anterior resection (LAR) among patients with stage II/III rectal cancer.

Methods. In this comparative-effectiveness study, we built Markov microsimulation models to simulate disease progression, death, costs, and quality-adjusted life-years (QALYs) for WW or APR/LAR. We assessed cost effectiveness using the incremental cost-effectiveness ratio (ICER), with ICERs under $\$ 100,000 /$ QALY considered cost effective. Probabilities of disease progression, death,
\end{abstract}

Christina Liu Cui and William Yu Luo contributed equally to this work.

(C) This is a U.S. government work and not under copyright protection in the U.S.; foreign copyright protection may apply 2021

First Received: 20 August 2020

Accepted: 22 June 2021;

Published Online: 16 September 2021

N. Lopez, MD, MAS

e-mail: nelopez@health.ucsd.edu and health utilities were extracted from published, peerreviewed literature. We assessed costs from the payer perspective.

Results. WW dominated both LAR and APR at a willingness to pay (WTP) threshold of $\$ 100,000$. Our model was most sensitive to rates of distant recurrence and regrowth after WW. Probabilistic sensitivity analysis demonstrated that WW was the dominant strategy over both APR and LAR over $100 \%$ of iterations across a range of WTP thresholds from $\$ 0-250,000$.

Conclusions. Our study suggests WW could reduce overall costs and increase effectiveness compared with either LAR or APR. Additional clinical research is needed to confirm the clinical efficacy and cost effectiveness of WW compared with surgery in rectal cancer.

Over 700,000 cases of colorectal cancer are diagnosed annually worldwide, with $30 \%$ of tumors arising in the rectum. ${ }^{1,2}$ One-third of rectal cancer patients present with locally advanced disease. ${ }^{3}$ Current standard of care for locally advanced rectal cancer consists of neoadjuvant radiation, with or without chemotherapy, followed by radical resection. ${ }^{4}$ Extent of resection, low anterior resection (LAR) or abdominoperineal resection (APR), depends on tumor relation to the sphincter complex.

However, evidence suggests that some patients may be able to avoid surgery. Data from prospective clinical trials demonstrate that up to $25 \%$ of patients have a pathological complete response (pCR; no viable tumor on pathological examination) after neoadjuvant chemoradiation. ${ }^{5-8}$ These patients have demonstrated lower odds of local or distant 
recurrence and greater odds of 5-year disease-free survival. ${ }^{5,9,10}$ Given the significant decrease in quality of life associated with surgery, surgeons are increasingly considering whether patients with pCR may represent a cohort that could have avoided surgery. ${ }^{11,12}$ However, since pCR can only be determined after surgical resection, postneoadjuvant selection criteria for patients typically relies on clinical complete response (cCR). While an imperfect approximation, cCR can act as a surrogate marker for $\mathrm{pCR}$, and is determined using post-chemoradiation clinical, endoscopic, or radiographic evaluations. Watch and wait (WW) protocols for patients with cCR have gained increasing popularity since described by Habr-Gama et al. in 2004. ${ }^{13}$ Patients with cCR undergoing a WW protocol have approximately $10 \%$ risk of distant recurrence and $20 \%$ risk of local regrowth; ${ }^{14,15}$ the majority of patients with regrowth can undergo salvage surgery $(95 \%){ }^{14,16}$ Similarly, when comparing all patients undergoing surgery with all patients managed with the WW strategy, WW patients demonstrate non-inferior local recurrence/regrowth-free survival. ${ }^{17}$ These data indicate that WW is safe and feasible for patients with cCR after neoadjuvant therapy. This is true even despite the heterogeneity of staging and surveillance strategies used in current data. ${ }^{15,18}$

WW protocols may also confer higher patient quality of life. Increased colostomy-free survival, as demonstrated by Renehan et al., is a perceived benefit among patients eligible for the WW treatment approach. ${ }^{17}$ This is also supported by a cross-sectional study by the Dutch Prospective Data Collection Initiative on Colorectal Cancer (PLCRC), in which WW was preferred by the majority of patients. Indeed, WW had one of the highest perceived health utility scores out of all interventions. ${ }^{19}$ In comparison, surgical intervention has well-described long-term negative impacts on patient quality of life, with patients undergoing LAR reporting an approximately 17\% decrease in health utility during the first few months after surgery. ${ }^{20}$ Despite the fact that patient quality of life can be greatly impacted by surgery, helping patients consider strategies for optimizing quality of life in medical decision-making processes can often fall short. ${ }^{21-23}$

In this study, we aimed to compare quality of life (as measured in quality-adjusted life-years [QALYs]), efficacy, and cost of treatment among patients undergoing WW versus LAR or APR.

\section{METHODS}

\section{Cost-Effectiveness Model}

We created Markov microsimulation models for patients with cCR after neoadjuvant chemoradiation for locally advanced rectal cancer. The models simulate the outcomes of 10,000 patients with stage II/III rectal cancer with a cCR after neoadjuvant chemoradiation opting for WW or radical surgery. Given the different quality of life associated with APR and LAR, we created separate models for each surgical option (WW versus APR, and WW versus LAR). The models included six distinct heath states: stable disease, local progression with salvage surgery, local progression without salvage surgery, distant progression, both local and distant progression, and death. Our local and distant progression health state allowed us to model concurrently diagnosed local and distant recurrence, local recurrence with subsequent finding of distant recurrence, and distant recurrence with subsequent findings of local recurrence. Each of these events had distinct transition probabilities associated with them (Table 1). Patients all started in the 'stable disease' state in either the WW or initial surgery arms and could stay in this state, transition to one of the progression states, or die (Fig. 1). Patients could die from cancer-related or non-cancer causes.

\section{Base-Case Description}

The standard (base-case) patient analyzed was a patient with locally advanced (T3, N Any or T1-2, N1-2) rectal cancer who achieved a complete clinical response following chemoradiation. To make the $\mathrm{WW}$ and LAR/APR groups comparable, we included only locally advanced (T3, N Any or T1-2, N1-2) disease. Therefore, all patients would undergo neoadjuvant chemoradiation. Based on National Comprehensive Cancer Network (NCCN) guidelines, all these patients were modeled to undergo adjuvant therapy to total 6 months' duration of therapy. Given 11/2 months of neoadjuvant chemoradiation, we modeled this as 4 months of adjuvant chemotherapy to start postoperatively, when patients entered the well state ( 2 months after resection). The base case for APR received permanent ostomies. LAR patients were modeled to have ostomies for 6 months postoperatively (4 months of chemotherapy + 2 months of recovery prior to ostomy reversal). All patients undergoing salvage for local recurrence/regrowth received APR or LAR, depending on the model.

In contrast to patients undergoing APR or LAR, patients monitored under WW protocols were modeled to directly enter the stable disease state for post-neoadjuvant treatment surveillance based on previously published Memorial Sloan-Kettering nonoperative management surveillance programs. ${ }^{24} \mathrm{WW}$ patients could then remain with no evidence of disease or develop a potentially resectable local regrowth. Patients who underwent resection for local regrowth were then assigned to a postoperative stable disease state, in which they could then have a potentially salvageable local recurrence. We adopted this approach in 


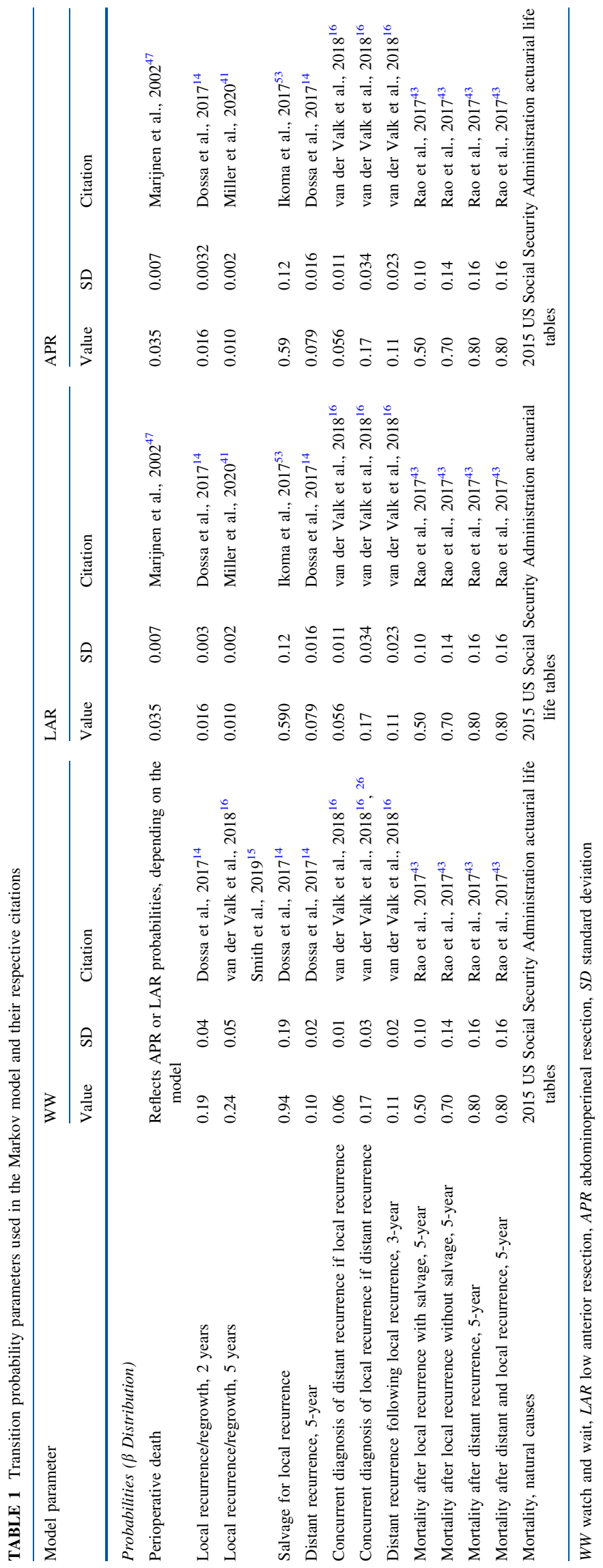


FIG. 1 Transition state diagram summarizing the Markov simulation model employed in our study. $L A R$ low anterior resection, $A P R$ abdominoperineal resection

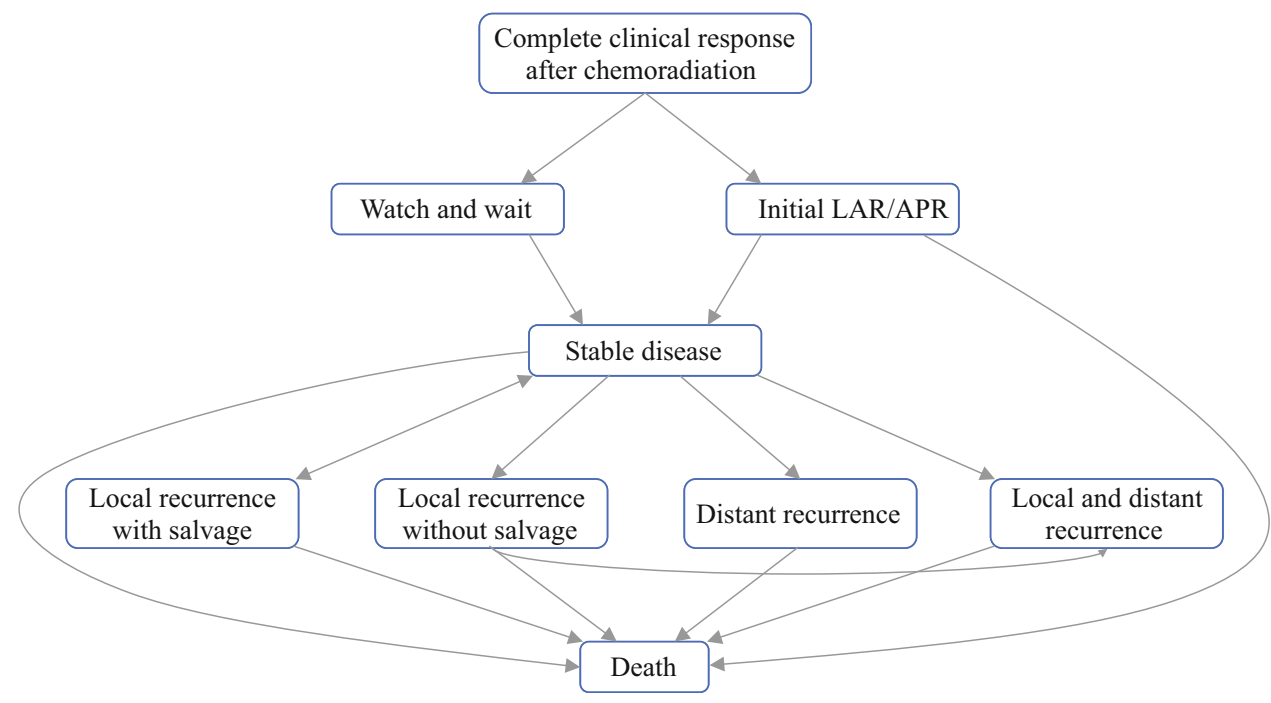

modeling the post-neoadjuvant treatment course for WW patients to reflect the reality that WW does not preclude surgery, but rather offers patients an opportunity to avoid resection, reserving radical resection for local regrowth.

The model used a 1-month cycle length and extended over a 5-year time horizon. We used a 5-year time horizon because the majority $(80 \%)$ of disease recurrences occur within 2 years of resection and/or treatment, with over $98 \%$ of recurrences falling within 5 years. ${ }^{25}$ Most of the available literature comparing WW versus radical resection is also limited to 5-year follow-up, thus limiting any attempt to expand our time horizon beyond 5 years without making assumptions or extrapolating data. ${ }^{14}$

We performed all model building and analysis using TreeAge Pro Healthcare version 2021 (Williamstown, MA, USA).

\section{Model Parameters}

Probabilities of disease progression and cancer death for all arms in the study were determined from peer-reviewed sources (Table 1). Probability of death from natural causes was determined from Social Security life tables (Table 1). Effectiveness was measured in QALYs, which reflects the product of health utility and time. Health utility represents patient quality of life (measured in QALYs), which ranges from 0 (death) to 1 (perfect health). Each health state has a distinct health utility score, and the health utility after surgery depended on the presence or absence of an ostomy (Table 2).
Costs were determined from the payer perspective using Current Procedural Terminology (CPT) billing codes and the 2019 Physician's Fee Schedule, and were adjusted to 2019 US dollars (\$) using the Consumer Price Index to account for inflation. ${ }^{26}$ To more accurately reflect costs of cancer care, we incorporated time-dependent costs for the following components of treatment: chemotherapy, ostomy care, and post-treatment cancer surveillance costs. An exception to this was the cost of APR/LAR. This was because of the relative heterogeneity of CPT billing codes used to document such operations. Moreover, reliance on CPT billing codes does not reflect the varied experiences in anesthesia and inpatient admissions. To ensure a more standard reporting of operative cost, we used a previously published source instead of the Consumer Price Index. ${ }^{27}$ The same source was used to determine the costs of adjuvant chemotherapy. Surveillance after surgery was based on the American Society of Clinical Oncology guidelines $^{28}$ (Table 3).

\section{Example of Transition Through Model}

A base-case patient randomized to surgery may have stable disease for 1 year until they develop local recurrence not eligible for surgical resection. Within the model, this patient would cycle 12 times (one cycle/month) within the stable disease health state then progress to local recurrence without salvage. This patient may then develop distant recurrence 6 months later. Within the model, this patient would cycle within the local recurrence without salvage state for six cycles (i.e. months) before progressing to the 


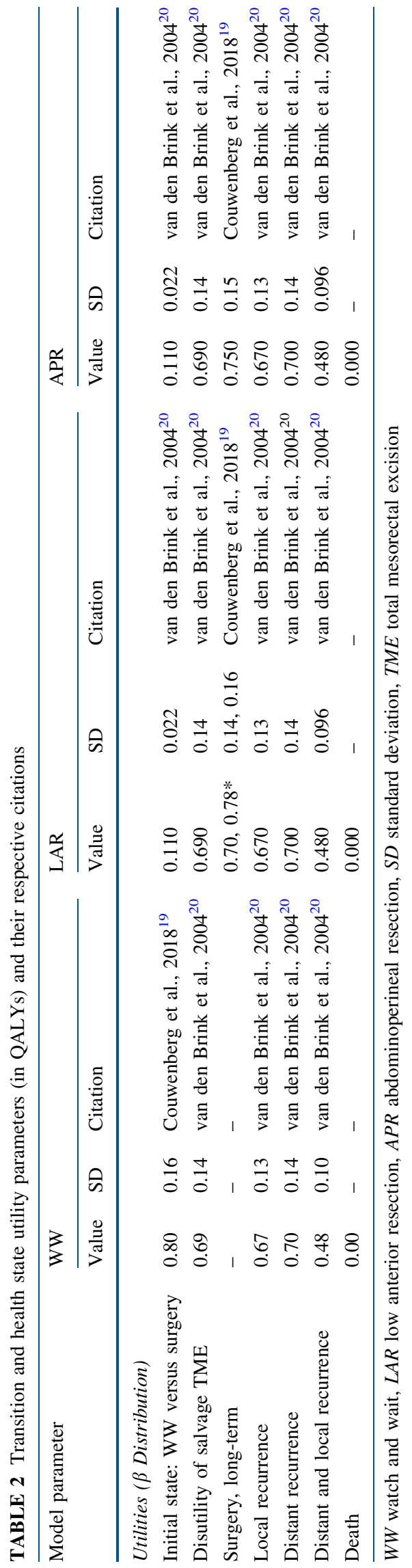

local and distant recurrence stage. As the patient cycles through each health state, there is a chance of death from natural causes, a chance to remain in said health state, or a chance to transition to a different health state. Each transition and health state has an associated cost (\$) and utility (QALYs) that accumulates as a patient progresses through the model until the time horizon or death is reached.

\section{Statistical Analysis}

Cost effectiveness was assessed with an incremental cost-effectiveness ratio (ICER), which represents the incremental costs divided by incremental QALYs of each treatment group. ICERs under \$100,000/QALY were considered cost effective. The willingness-to-pay (WTP) threshold is defined as the ICER below which an intervention is considered cost-effective. We used $\$ 100,000$ as the threshold based on previously published literature as well as other peer-reviewed cost-effectiveness analyses. $^{29-31}$ Treatments that lowered costs and increased effectiveness were considered dominant. All costs and utilities were discounted by $3 \%$ annually with half-cycle corrections. We conducted one-way (i.e. deterministic) sensitivity analysis on all probabilities, utilities, and costs to determine their impact on cost effectiveness. For transition probabilities and utilities in our one-way sensitivity analysis, we used the broadest range of values possible (i.e. as close to $0-100 \%$ ), and for cost parameters, we used a lower and upper bound of 5\% and 195\% the base cost parameter, respectively. We conducted probabilistic sensitivity analysis where we modeled transition probabilities and health utilities with $\beta$ distributions, and costs with $\gamma$ distributions. We identified standard deviations for each variable distribution from the literature and used a standard deviation equal to $20 \%$ of the mean with unknown standard deviations. We tested different values of our unknown standard deviation (ranging from $10 \%$ to $40 \%$ of the mean) in a sensitivity analysis, which did not impact our results (analysis not shown). This study was conducted and published according to previously reported guidelines. ${ }^{32}$

\section{Human Subjects}

We did not use data generated directly from human subjects in our work.

\section{RESULTS}

\section{Base-Case Microsimulation}

In our APR versus WW base-case microsimulation, APR cases had a slightly higher 5-year overall mortality 


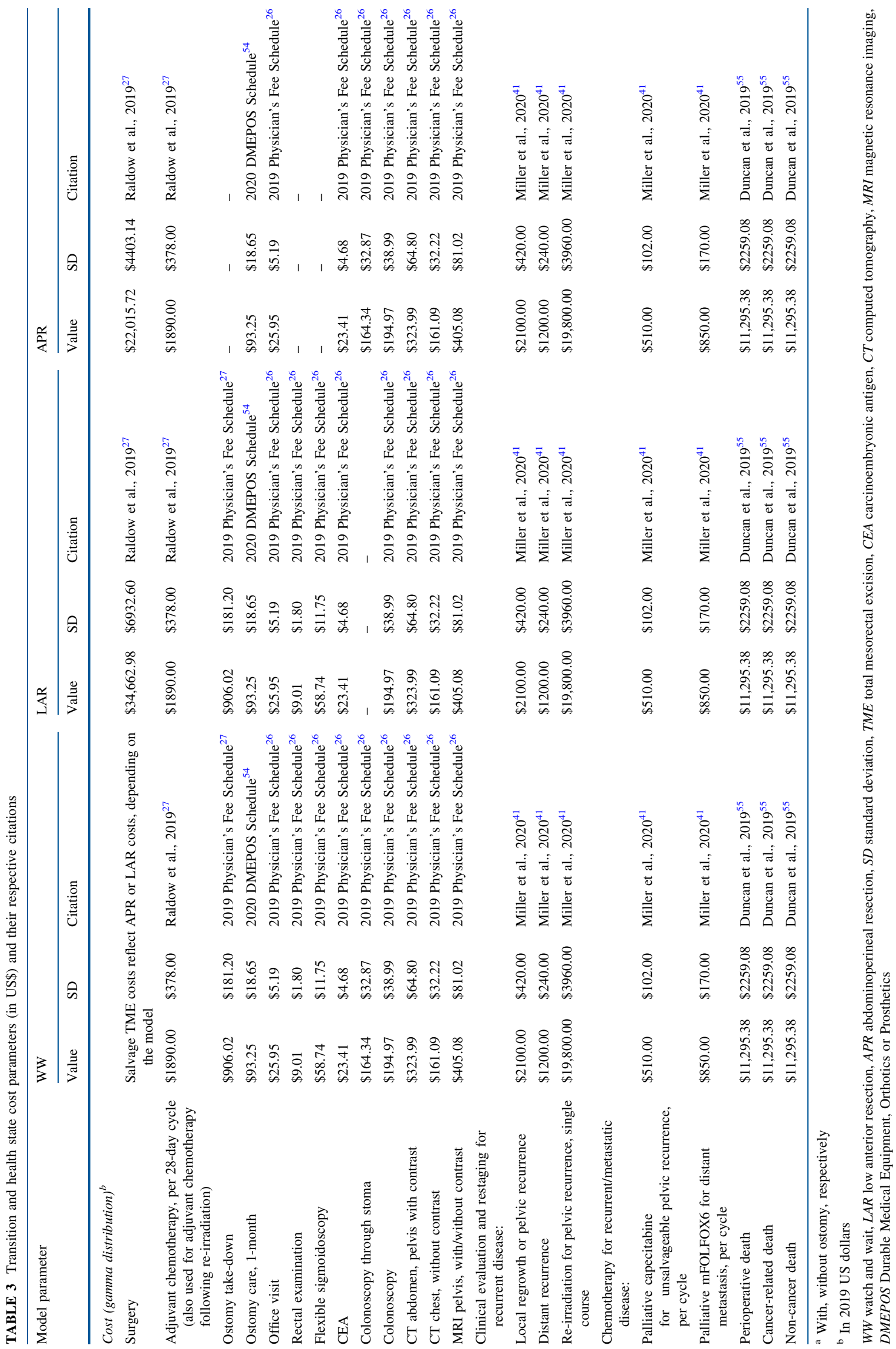


TABLE 4 Five-year survival and recurrence outcomes of the base-case analysis

\begin{tabular}{lrrrr}
\hline & APR & WW & LAR & WW \\
\hline Recurrence, 5-year & & & & \\
Local regrowth (during the WW period) & - & 31.25 & - & 30.83 \\
Local recurrence (following first operation) & 3.69 & 2.33 & 3.37 & 2.37 \\
Distant only & 7.52 & 10.32 & 7.40 & 10.37 \\
Local and distant (includes concurrent local regrowth if WW) & 1.46 & 2.93 & 1.33 & 3.07 \\
Mortality, 5-year & & & & \\
Overall & 14.71 & 13.71 & 14.79 & 13.22 \\
Cancer-specific & 4.69 & 6.14 & 4.48 & 5.99 \\
\hline
\end{tabular}

Data are expressed as percentages

$W W$ watch and wait, $L A R$ low anterior resection, $A P R$ abdominoperineal resection

\begin{tabular}{lllrr}
\hline & APR & WW & LAR & \multicolumn{1}{c}{ WW } \\
\hline Cost & $\$ 40,655.13$ & $\$ 23,894.94$ & $\$ 50,484.77$ & $\$ 26,499.86$ \\
Effectiveness (QALY) & 3.17 & 3.40 & 3.24 & 3.41 \\
Incremental cost (vs. WW) & $\$ 16,760.63$ & & $\$ 23,984.91$ & \\
Incremental effectiveness (vs. WW) & -0.23 & & -0.17 & \\
ICER (\$/QALY) & Dominated & & Dominated & \\
\hline
\end{tabular}

$W W$ watch and wait, $L A R$ low anterior resection, $A P R$ abdominoperineal resection, $Q A L Y$ quality-adjusted life-year, ICER incremental cost-effectiveness ratio
TABLE 5 Outcomes of the base-case analysis
QALYs compared with APR (Table 5). By reducing costs and increasing effectiveness, WW dominated both LAR and APR.

\section{Deterministic Sensitivity Analysis}

We found that both microsimulations were sensitive to 2-year local regrowth rates following WW, 5-year distant recurrence rates following WW, and WW utility. In addition, our APR versus WW microsimulation was sensitive to APR utility, and our LAR versus WW microsimulation was sensitive to LAR utility following ostomy reversal (Table 6). Specifically, both APR and LAR became more cost-effective than WW if 2-year local recurrence rates following WW exceeded 86 and $83 \%$, respectively. Similarly, APR and LAR were more cost-effective than WW when 5-year distant recurrence rates exceeded 57 and 56\%, respectively. APR was more cost effective than WW when utility after APR was higher than for WW. Similarly, LAR was more cost effective when utility after LAR and ostomy reversal was higher than for WW.

Despite some evidence suggesting that delaying adjuvant treatment past 4 weeks was detrimental to outcomes, timing of chemotherapy initiation ranged between 4 and 12 weeks among published randomized trials. ${ }^{33-38}$ We thus performed a sensitivity analysis of this parameter. For each 


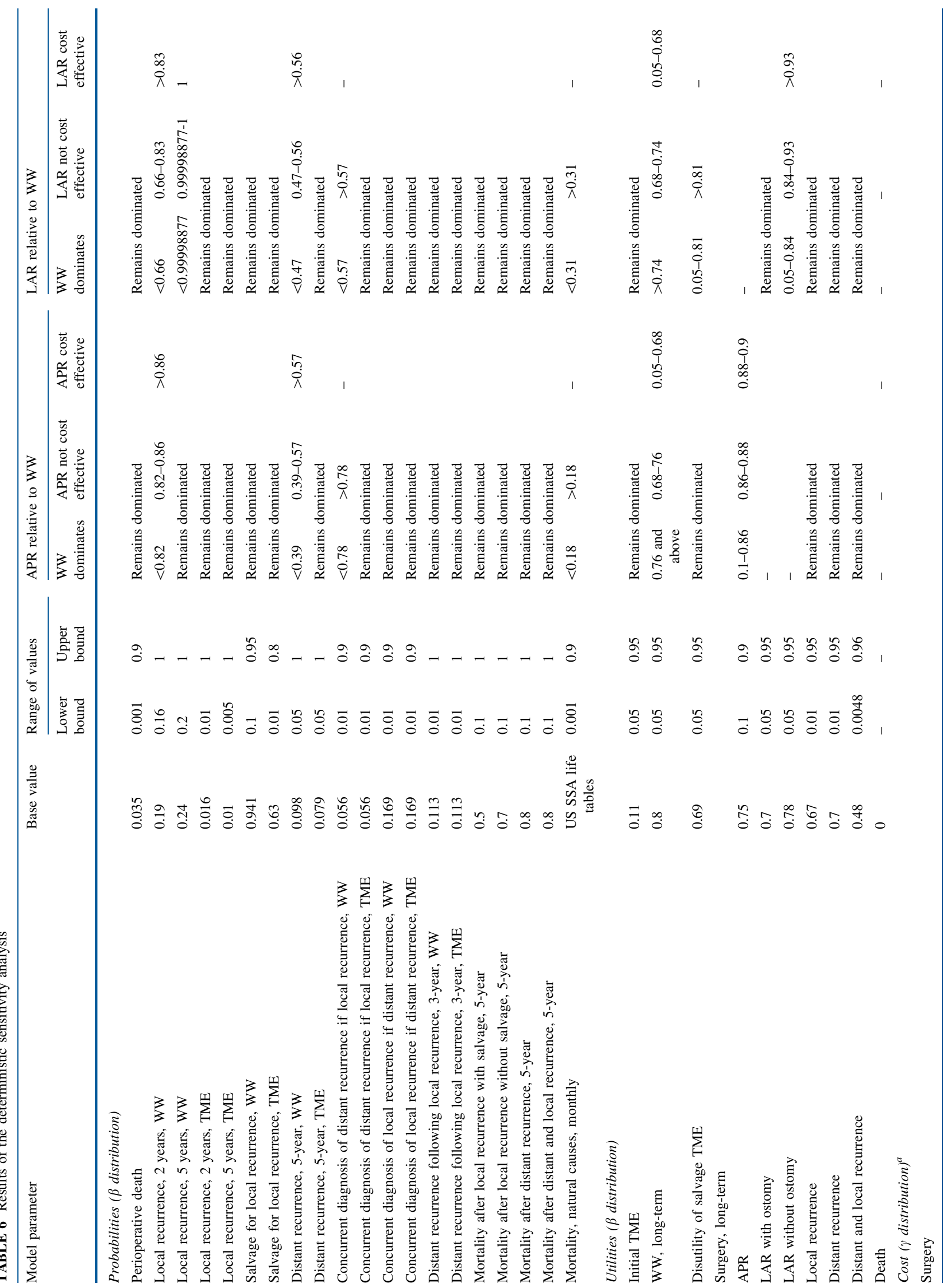




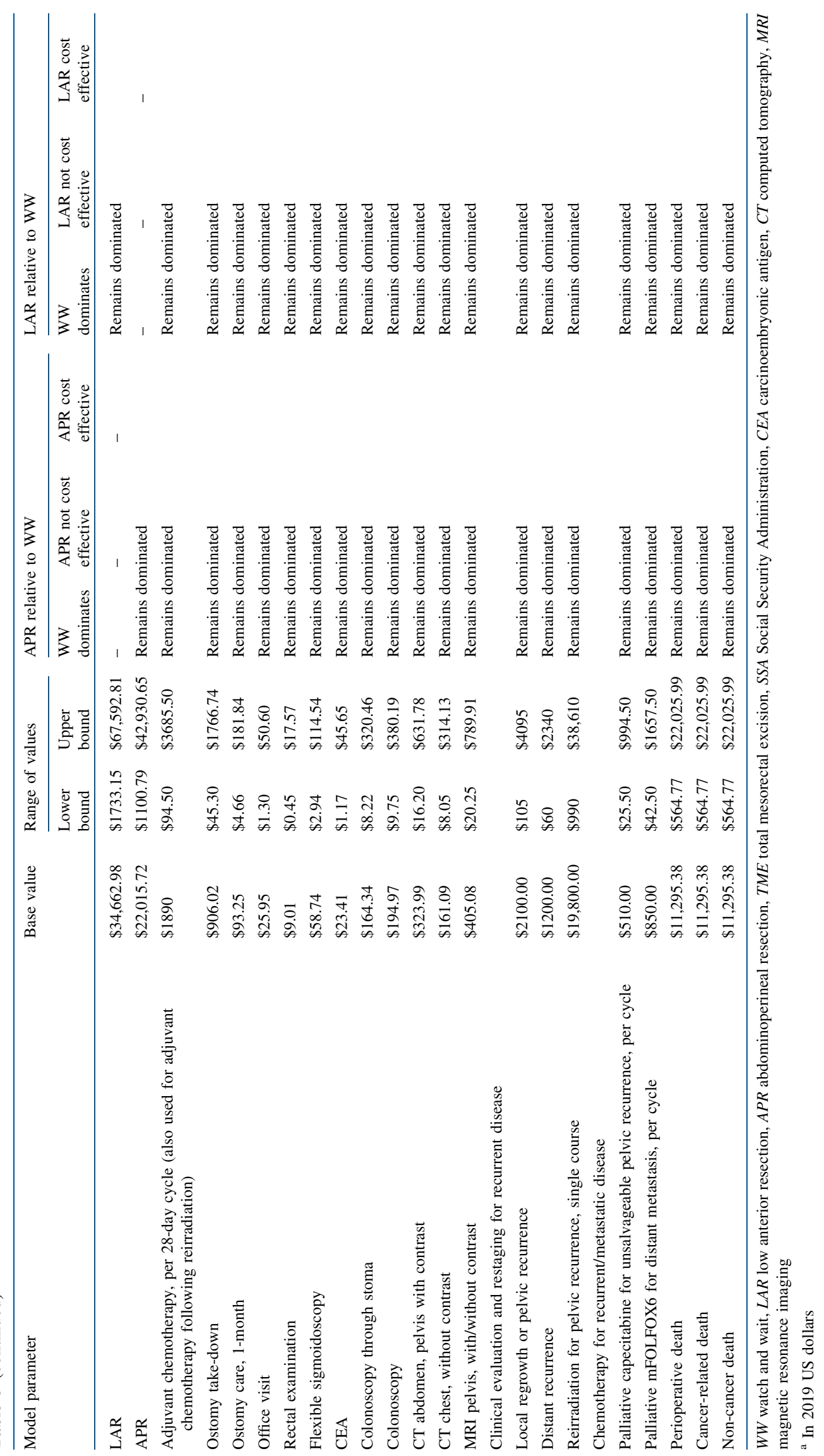


tree, we modeled adjuvant chemotherapy to start at 4,8 , and 12 weeks post-intervention. In all scenarios, WW remained dominant over LAR and APR.

\section{Probabilistic Sensitivity Analysis}

Our probabilistic sensitivity analysis (100 microsimulations of 10,000 cases) for both LAR and APR models versus WW found that WW was dominant or was considered cost effective over both strategies across a range of WTP thresholds from \$0-\$200,000/QALY in 100\% of the iterations tested.

\section{DISCUSSION}

We used two separate Markov models, one comparing APR with WW, and one comparing LAR with WW, to simulate 10,000 cases per model. At a WTP threshold of $\$ 100,000$, WW was the dominant strategy over both LAR and APR. In fact, even when increasing WTP thresholds to $\$ 250,000$, the WW strategy remained dominant. Thus, WW was significantly more cost effective than resection.

Both APR and LAR models indicated that surgery was associated with increased 5-year mortality but decreased cancer-specific mortality. Overall, the findings suggest that patients undergoing surgery, regardless of type, seem to pay a mortality penalty (unrelated to cancer) that is greater in magnitude than the increased cancer-specific mortality associated with WW. These treatment-based differences can logically be attributed to both the risks associated with surgery and the marginal increased risk of leaving residual cancer in place with the WW strategy.

Based on these data, we suggest considering the WW strategy for patients with cCR; however, we must also acknowledge the effects on distant recurrence. Both distant metastases, and combined local and distant metastases, were slightly more common in patients treated with WW. This observation, demonstrating increased metastatic potential when the primary tumor is left in situ, is consistent with a priming role for the primary tumor, where the primary tumor sends signals to distant sites to prepare them for metastatic seeding. ${ }^{39}$ However, this supposes remnant tumor tissue and it is unclear whether tissue recently cleared of malignancy might assume a similar role.

While these numbers are interesting to examine, they are principally meant to confirm that the model functions as expected. Thus, we must also determine whether our findings might be affected by factors built into the model. Both models were sensitive to regrowth rates, distant recurrence, and utilities associated with WW or following surgery. In both models, cost effectiveness of WW was sensitive to rates of regrowth and distant recurrence after
WW. From a clinical standpoint, most hesitation to embrace WW stems from the possibility that the strategy may put patients at higher risk of developing unresectable or distant metastatic disease. In the APR versus WW model, the 2-year local regrowth rate during WW would have to be $>86 \%$ to make APR more cost effective than WW. Similarly, a threshold above 90\% 2-year local regrowth after WW would result in LAR being more cost effective. Both thresholds are much higher than the maximum $30 \%$ regrowth reported in the literature. ${ }^{14,16,40}$ The extreme nature of these thresholds may be due to the high salvage rates that we modeled, taken from the current literature. We also found that surgery (either LAR or APR) was only more cost effective if rates of 5-year distant metastatic disease exceeded $57 \%$ in the WW group. Again, this theoretical threshold is much higher than accepted distant recurrence rates of up to $13 \% .^{14}$ Therefore, from a cost-effectiveness perspective, WW is superior to both LAR and APR when clinically relevant regrowth and distant metastatic recurrence rates are considered.

Of note, our models were not sensitive to cost. Although various surveillance schedules after WW have been cited in literature, we did not vary the surveillance schedule in our model, which was based on just one of several published WW protocols. ${ }^{28}$ Altering the surveillance schedule might affect this result; however, we expect these results are robust and would remain unaltered since the model was not sensitive to cost in any capacity. Additionally, other groups who have approached modeling the data with various strategies, have arrived at a similar conclusion regarding cost. $^{41}$

Theoretically, APR and LAR could be more cost effective than WW if patient-reported quality of life following radical surgery was better than after $\mathrm{WW}^{19}$ (Table 6). However, these criteria are clinically unrealistic; we would rarely expect a patient who has had only chemoradiation to report worse quality of life than a patient who has had chemoradiation followed by radical surgery and possibly a permanent stoma. This is supported by literature gathered regarding patient-reported quality of life, which have consistently ranked perceived quality of life to be higher following WW than either APR or LAR. ${ }^{19,20}$

While recurrence rates are a priority among providers, patient-centered outcomes, such as QALYs, are crucial to acknowledge as well. Our model showed that implementing WW for rectal cancer patients with cCR after neoadjuvant therapy did not lead to an appreciable overall survival trade-off compared with either LAR or APR. However, WW had higher rates of local regrowth and marginally increased distant recurrence than both LAR and APR (Table 4). Despite this, WW offered a QALY and cost benefit compared with either operative approach. This is likely because cost-effectiveness analyses utilize a 
holistic approach balancing clinical outcomes, patient perspectives, and costs in a way that most studies focused on a single facet of patient care cannot offer.

Because WW dominated both LAR and APR in our base-case analyses, we did not expect WTP thresholds to significantly change our results. However, given the inherently subjective nature of WTP thresholds, we performed a probabilistic sensitivity analysis to test our model against a wide range of WTP thresholds $(\$ 0-\$ 200,000$ / QALY), which showed that both LAR and APR remained dominated by WW independent of the WTP threshold.

Few cost-effectiveness analyses on WW versus radical surgery have been published in the past decade. Neuman et al. conducted the first analyses in 2009 and their model found surgery to be cost effective compared with WW. ${ }^{42}$ While informative, their model was built on older publications, including studies with relatively small patient numbers and using expert opinion for critical values such as recurrence rates and health utilities. In contrast, Rao et al. and Smith et al. published a similar analysis in 2015 and 2017, respectively, that found WW to be cost effective compared with radical surgery; ${ }^{43,44}$ however, these results were specific to elderly patients and, once more, used numbers based on expert opinion alone. Moreover, neither study stratified analysis by type of operation (i.e. LAR versus APR), which has clear clinical effects on postoperative morbidity, mortality, and quality of life. ${ }^{45,46}$

The most recently published cost-effectiveness analysis by Miller et al. also concluded that WW was the dominant strategy compared with radical surgery. ${ }^{41}$ One difference between our models is our perioperative death parameter. While Miller et al. cite a perioperative mortality rate of $0.6 \%$, we modeled a rate of $3.5 \%$. Our parameter value is derived from a prospective multicenter randomized trial studying locally advanced rectal cancer patients in whom neoadjuvant therapy was an inclusion criterion. ${ }^{47}$ In contrast, Miller et al. used a more recent retrospective study that included all rectal cancer patients whether or not they received neoadjuvant treatment. ${ }^{48}$ Furthermore, while the newer ROLARR trial may have provided lower perioperative mortality risks, less than half of their cohorts received neoadjuvant treatment. ${ }^{49}$ Nonetheless, a sensitivity analysis, accounting for extreme ranges in perioperative mortality (from $0.1 \%$ to $90 \%$ ), showed that the perioperative mortality rate did not impact our results, therefore we maintained our analysis using mortality according to Marijnen et al. (Table 6). ${ }^{47}$

To complement and expand upon these models, we built two different models to capture outcomes unique to APR and LAR. In comparison, the model employed by Miller et al. permitted all patients the option of undergoing WW, LAR, or APR. However, APR is primarily reserved for rectal tumors that cannot be resected with an adequate margin using a sphincter-sparing approach, therefore patients who can undergo LAR and those who require an APR may have inherently different, preoperatively determined disease profiles, which might affect the cost effectiveness. ${ }^{50}$ As such, we believed two separate models were necessary. We also used a microsimulation, which, as discussed previously, allowed us to mimic the progress of individual patients. In addition, we allowed WW patients the opportunity to undergo two salvage surgeries (allowing for initial resection after regrowth as well as resection after recurrence if that occurred) as opposed to just one surgical intervention in the study by Miller et al. Our model also built-in greater granularity for regrowth/recurrence by allowing patients to experience both local regrowth and distant recurrence with different probabilities if distant recurrence was diagnosed first, and vice versa. ${ }^{16}$

These added intricacies can be attributed to our interdisciplinary approach to building and validating the model. Other differences include variations in sources of probabilities and utilities; we used the highest level of evidence from recently published data in well-established journals. ${ }^{14-16,19}$ All model utilities were drawn from an updated prospective survey by the PLCRC, which included more granular results. ${ }^{19}$ In contrast, prior cost-benefit analyses relied on less precise, prospective utility surveys, or on utilities for other disease processes such as prostate cancer. $^{43}$

Limitations to this study are inherent to any cost-effectiveness analysis. Intrinsic to their nature, models simplify complex diseases and processes involving outcomes, patient perspectives, access to care, and other social factors. For example, we were also unable to evaluate the impact of patient compliance on surveillance and treatment due to the lack of robust studies with details on real-world follow-up. These effects may be especially important within the WW protocol given the need for regular followup, although some data suggest that differences in compliance may not affect patient outcomes. ${ }^{17}$ Still, since we used real-world data from retrospective studies to build our models, impacts of patient compliance may be inherent in the transition probabilities we used. Other studies have also suggested that adherence to post-operative chemotherapy is lower than expected. ${ }^{51}$ In contrast, our model assumed that all patients undergoing surgery also underwent adjuvant chemotherapy. Our goal was to have the model align as closely as possible to the standard of care and was also built upon the precedence set by prior decision models on a similar topic. ${ }^{43}$ Despite this, our assumption of perfect adherence to adjuvant chemotherapy is made across all interventions in our models: LAR, APR, and WW, thus controlling for any potential confounding that treatment adherence may confer. 
Our reliance on costs from a payor perspective may appear to discount the very real costs experienced by patients. However, we stress that our health utilities, as measured in QALYs as outlined in our Methods section, are a useful surrogate for measuring the impacts (financial or otherwise) that diseases and their treatments have on our patients. Indeed, one can argue that financial impacts have real consequences on a patient's perceived quality of lifea metric that is captured by the QALYs documented in the current literature. ${ }^{19}$ Moreover, given the heterogenous nature of healthcare reimbursement in the US, costs from the payor (i.e. Medicare) perspective are often the best available standard measure. ${ }^{52}$

Additionally, models are only as accurate as the studies used to build them. The decision to pursue surgery will have long-term ramifications on patient survival and quality of life, but our model ended at 5 years due to the lack of robust follow-up data beyond then. Despite these limitations, we included the most granular and high-level data available, and our model was validated by an interdisciplinary team all regularly involved in the care for this patient population.

Lastly, this model is not intended to predict treatment outcomes (i.e. recurrence rates or mortality) for this patient population. Our goal is to understand how treatment options for this specific patient population differ from a cost and quality-of-life perspective. As such, external validation is not standard for cost-effectiveness modeling. Nonetheless, we found that our model outcomes for recurrence rates and mortality were comparable with those in the current literature. Ultimately, a randomized clinical trial will be needed to verify the clinical outcomes of each treatment approach. One such trial, NORWAIT, is currently underway in Norway (NCT03402477).

\section{CONCLUSIONS}

The results of our analyses suggest WW confers superior QALYs at lower costs when compared with radical surgery for patients with cCR after neoadjuvant chemoradiation for locally advanced rectal cancer. Based on our model, these benefits do not come at the expense of reduced overall survival. The WW strategy requires attention to detail in patient selection and unyielding diligence in surveillance. However, for eligible patients, it is a cost-saving approach that offers significantly improved quality of life without compromising oncologic outcomes.

ACKNOWLEDGMENT Work performed by CLC and WYL was supported in part by the National Institutes of Health award no. 1TL1TR001443. NEL was supported in part by the National Library of Medicine grant no. 5T15LM011271. SE and JM have received consultancy fees from entities unrelated to this work. BCC and SE have received fees for expert testimony from various law firms unrelated to this work. Daniel Simpson and Sonia Ramamoorthy have declared no conflicts of interest.

AUTHORS CONTRIBUTION Samuel Eisenstein: Consultant for Ethicon Surgical Robotics and Takeda. James Murphy: Receives compensation for consulting from Boston Consulting Group. Nicole Lopez: NLM grant for postdoctoral fellowship.

FUNDING This work was supported in part by the National Institutes of Health and National Library of Medicine.

DISLCOSURE Christina Liu Cui, William Yu Luo, Bard Clifford Cosman, Daniel Simpson, and Sonia Ramamoorthy have no disclosures to declare.

OPEN ACCESS This article is licensed under a Creative Commons Attribution 4.0 International License, which permits use, sharing, adaptation, distribution and reproduction in any medium or format, as long as you give appropriate credit to the original author(s) and the source, provide a link to the Creative Commons licence, and indicate if changes were made. The images or other third party material in this article are included in the article's Creative Commons licence, unless indicated otherwise in a credit line to the material. If material is not included in the article's Creative Commons licence and your intended use is not permitted by statutory regulation or exceeds the permitted use, you will need to obtain permission directly from the copyright holder. To view a copy of this licence, visit http://creativecommons. org/licenses/by/4.0/.

\section{REFERENCES}

1. Rawla P, Sunkara T, Barsouk A. Epidemiology of colorectal cancer: incidence, mortality, survival, and risk factors. Prz Gastroenterol. 2019;14(2):89-103. https://doi.org/10.5114/pg.2018. 81072.

2. Feeney G, Sehgal R, Sheehan M, et al. Neoadjuvant radiotherapy for rectal cancer management. World $J$ Gastroenterol. 2019;25(33):4850-69. https://doi.org/10.3748/wjg.v25.i33.4850.

3. Siegel RL, Miller KD, Jemal A. Cancer statistics, 2019. $C A$ Cancer J Clin. 2019;69(1):7-34. https://doi.org/10.3322/caac. 21551.

4. Monson JRT, Weiser MR, Buie WD, et al. Practice parameters for the management of rectal cancer (revised). Dis Colon Rectum. 2013;56(5):535-50. https://doi.org/10.1097/DCR.0b013e31828cb $66 \mathrm{c}$.

5. Maas M, Nelemans PJ, Valentini V, et al. Long-term outcome in patients with a pathological complete response after chemoradiation for rectal cancer: a pooled analysis of individual patient data. Lancet Oncol. 2010;11(9):835-44. https://doi.org/10.1016/ S1470-2045(10)70172-8.

6. Smith FM, Waldron D, Winter DC. Rectum-conserving surgery in the era of chemoradiotherapy. $B r \quad J$ Surg. 2010;97(12):1752-64. https://doi.org/10.1002/bjs.7251.

7. Erlandsson J, Lorinc E, Ahlberg M, et al. Tumour regression after radiotherapy for rectal cancer - Results from the randomised Stockholm III trial. Radiother Oncol. 2019;135:178-86. https://d oi.org/10.1016/j.radonc.2019.03.016.

8. Cisel B, Pietrzak L, Michalski W, et al. Long-course preoperative chemoradiation versus 5 x 5 Gy and consolidation chemotherapy for clinical T4 and fixed clinical T3 rectal cancer: long-term results of the randomized Polish II study. Ann Oncol. 2019;30(8):1298-303. https://doi.org/10.1093/annonc/mdz186. 
9. Yeo SG, Kim DY, Kim TH, et al. Pathologic complete response of primary tumor following preoperative chemoradiotherapy for locally advanced rectal cancer: long-term outcomes and prognostic significance of pathologic nodal status (KROG 09-01). Ann Surg. 2010;252(6):998-1004. https://doi.org/10.1097/SLA.0 b013e3181f3f1b1.

10. Martin ST, Heneghan HM, Winter DC. Systematic review and meta-analysis of outcomes following pathological complete response to neoadjuvant chemoradiotherapy for rectal cancer. $\mathrm{Br}$ J Surg. 2012;99(7):918-28. https://doi.org/10.1002/bjs.8702.

11. Borowski DW, Bradburn DM, Mills SJ, et al. Volume-outcome analysis of colorectal cancer-related outcomes. $\mathrm{Br} J$ Surg. 2010;97(9):1416-30. https://doi.org/10.1002/bjs.7111.

12. Paun BC, Cassie S, MacLean AR, Dixon E, Buie WD. Postoperative complications following surgery for rectal cancer. Annals of surgery. 2010;251(5):807-18. https://doi.org/10.1097/SLA.0b 013e3181dae4ed.

13. Habr-Gama A, Perez RO, Nadalin W, et al. (2004) Operative versus nonoperative treatment for stage 0 distal rectal cancer following chemoradiation therapy: long-term results. Ann Surg. Oct ;240(4):711-7; discussion 717-8. doi:https://doi.org/10.1097/ 01.sla.0000141194.27992.32

14. Dossa F, Chesney TR, Acuna SA, Baxter NN. A watch-and-wait approach for locally advanced rectal cancer after a clinical complete response following neoadjuvant chemoradiation: a systematic review and meta-analysis. Lancet Gastroenterol Hepatol. 2017;2(7):501-13. https://doi.org/10.1016/s2468-1253( 17)30074-2.

15. Smith JJ, Strombom P, Chow OS, et al. Assessment of a watchand-wait strategy for rectal cancer in patients with a complete response after Neoadjuvant therapy. JAMA Oncol. 2019;5(4):e185896-e185896. https://doi.org/10.1001/jamaoncol. 2018.5896.

16. van der Valk MJM, Hilling DE, Bastiaannet E, et al. Long-term outcomes of clinical complete responders after neoadjuvant treatment for rectal cancer in the International Watch and Wait Database (IWWD): an international multicentre registry study. Lancet. 2018;391(10139):2537-45. https://doi.org/10.1016/S014 0-6736(18)31078-X.

17. Renehan AG, Malcomson L, Emsley R, et al. Watch-and-wait approach versus surgical resection after chemoradiotherapy for patients with rectal cancer (the OnCoRe project): a propensityscore matched cohort analysis. Lancet Oncol. 2016;17(2):174-83. https://doi.org/10.1016/S1470-2045(15)00467-2.

18. Pomerri F, Pucciarelli S, Maretto I, et al. Prospective assessment of imaging after preoperative chemoradiotherapy for rectal cancer. Surg. 2011;149(1):56-64. https://doi.org/10.1016/j.surg. 2010.03.025.

19. Couwenberg AM, Intven MPW, Burbach JPM, Emaus MJ, van Grevenstein WMU, Verkooijen HM. Utility scores and preferences for surgical and organ-sparing approaches for treatment of intermediate and high-risk rectal cancer. Dis Colon Rectum. 2018;61(8):911-9. 0000000000001029.

20. Van Den Brink M, Van Den Hout WB, Stiggelbout AM, et al. Cost-utility analysis of preoperative radiotherapy in patients with rectal cancer undergoing total mesorectal excision: a study of the Dutch Colorectal Cancer Group. $J$ Clin Oncol. 2004;22(2):244-53. https://doi.org/10.1200/JCO.2004.04.198.

21. Zafar SY, Alexander SC, Weinfurt KP, Schulman KA, Abernethy AP. Decision making and quality of life in the treatment of cancer: a review. Support Care Cancer. 2009;17(2):117-27. h ttps://doi.org/10.1007/s00520-008-0505-2.

22. Balch A. Talking about costs of care during shared decision making-Part one: the problem. J Clin Pathw. 2018;4(6):32-3. h ttps://doi.org/10.25270/jcp.2018.08.00031.
23. Guidet B, Beale R. Should cost considerations be included in medical decisions? Yes. Intensive Care Med. 2015;41(10):1838-40. https://doi.org/10.1007/s00134-015-39886.

24. Smith JJ, Chow OS, Gollub MJ, et al. Organ Preservation in Rectal Adenocarcinoma: a phase II randomized controlled trial evaluating 3-year disease-free survival in patients with locally advanced rectal cancer treated with chemoradiation plus induction or consolidation chemotherapy, and total mesorectal excision or nonoperative management. BMC Cancer. 2015;15:767. http s://doi.org/10.1186/s12885-015-1632-z.

25. Tan WJ, Tan HJ, Dorajoo SR, Foo FJ, Tang CL, Chew MH. Rectal cancer surveillance-recurrence patterns and survival outcomes from a cohort followed up beyond 10 years. J Gastrointest Cancer. 2018;49(4):422-8. https://doi.org/10.1007/s12029-017-9 984-z.

26. 83 Medicare Program; Revisions to Payment Policies Under the Physician Fee Schedule and Other Revisions to Part B for CY 2019; Medicare Shared Savings Program Requirements; Quality Payment Program; Medicaid Promoting Interoperability Program; Quality Payment Program-Extreme and Uncontrollable Circumstance Policy for the 2019 MIPS Payment Year; Provisions From the Medicare Shared Savings ProgramAccountable Care Organizations-Pathways to Success; and Expanding the Use of Telehealth Services for the Treatment of Opioid Use Disorder Under the Substance Use-Disorder Prevention That Promotes Opioid Recovery and Treatment (SUPPORT) for Patients and Communities Act. US Centers for Medicare \& Medicaid Services; 2018.

27. Raldow AC, Chen AB, Russell M, et al. Cost-effectiveness of short-course radiation therapy vs long-course chemoradiation for locally advanced rectal cancer. JAMA Netw Open. 2019;2(4):e192249. https://doi.org/10.1001/jamanetworkopen.20 19.2249 .

28. Meyerhardt JA, Mangu PB, Flynn PJ, et al. Follow-up care, surveillance protocol, and secondary prevention measures for survivors of colorectal cancer: American Society of Clinical Oncology clinical practice guideline endorsement. J Clin Oncol. 2013;31(35):4465-70. https://doi.org/10.1200/JCO.2013.50. 7442.

29. Kaplan RM, Bush JW. Health-related quality of life measurement for evaluation research and policy analysis. Health Psychol. 1982;1(1):61-80. https://doi.org/10.1037/0278-6133.1.1.61.

30. Neumann PJ, Cohen JT, Weinstein MC. Updating cost-effectiveness-the curious resilience of the $\$ 50,000$-per-QALY threshold. N Engl J Med. 2014;371(9):796-7. https://doi.org/10. 1056/NEJMp1405158.

31. Acevedo JR, Fero KE, Wilson B, et al. Cost-effectiveness analysis of elective neck dissection in patients with clinically nodenegative oral cavity cancer. J Clin Oncol. 2016;34(32):3886-91. https://doi.org/10.1200/JCO.2016.68.4563.

32. Sanders GD, Neumann PJ, Basu A, et al. Recommendations for conduct, methodological practices, and reporting of cost-effectiveness analyses: second panel on cost-effectiveness in health and medicine. JAMA. 2016;316(10):1093-103. https://doi.org/10. 1001/jama.2016.12195.

33. Collette L, Bosset JF, den Dulk M, et al. Patients with curative resection of cT3-4 rectal cancer after preoperative radiotherapy or radiochemotherapy: does anybody benefit from adjuvant fluorouracil-based chemotherapy? A trial of the European organisation for research and treatment of cancer radiation oncology group. J Clin Oncol. 2007;25(28):4379-86. https://doi. org/10.1200/JCO.2007.11.9685.

34. Bosset JF, Collette L, Calais G, et al. Chemotherapy with preoperative radiotherapy in rectal cancer. $N$ Engl J Med. 2006;355(11):1114-23. https://doi.org/10.1056/NEJMoa060829. 
35. Sainato A, Cernusco Luna Nunzia V, Valentini V, et al. No benefit of adjuvant Fluorouracil Leucovorin chemotherapy after neoadjuvant chemoradiotherapy in locally advanced cancer of the rectum (LARC): Long term results of a randomized trial (I-CNRRT). Radiother Oncol. 2014;113(2):223-9. https://doi.org/10.10 16/j.radonc.2014.10.006.

36. Breugom AJ, van Gijn W, Muller EW, et al. Adjuvant chemotherapy for rectal cancer patients treated with preoperative (chemo)radiotherapy and total mesorectal excision: a Dutch Colorectal Cancer Group (DCCG) randomized phase III trial. Ann Oncol. 2015;26(4):696-701. https://doi.org/10.1093/annonc/ mdu560.

37. Glynne-Jones R, Counsell N, Quirke P, et al. Chronicle: results of a randomised phase III trial in locally advanced rectal cancer after neoadjuvant chemoradiation randomising postoperative adjuvant capecitabine plus oxaliplatin (XELOX) versus control. Ann Oncol. 2014;25(7):1356-62. https://doi.org/10.1093/annonc/md u147.

38. Biagi JJ, Raphael MJ, Mackillop WJ, Kong W, King WD, Booth CM. Association between time to initiation of adjuvant chemotherapy and survival in colorectal cancer: a systematic review and meta-analysis. JAMA. 2011;305(22):2335-42. http s://doi.org/10.1001/jama.2011.749.

39. Peinado $\mathrm{H}$, Zhang $\mathrm{H}$, Matei IR, et al. Pre-metastatic niches: organ-specific homes for metastases. Nat Rev Cancer. 2017;17(5):302-17. https://doi.org/10.1038/nrc.2017.6.

40. Ayloor Seshadri R, Kondaveeti SS, Jayanand SB, et al. Complete clinical response to neoadjuvant chemoradiation in rectal cancers: can surgery be avoided? Hepatogastroenterol. 2013;60(123):410-4. https://doi.org/10.5754/hge12354.

41. Miller JA, Wang H, Chang DT, Pollom EL. Cost-effectiveness and quality-adjusted survival of watch-and-wait after complete response to chemoradiotherapy for rectal cancer. J Natl Cancer Inst. 2020. https://doi.org/10.1093/jnci/djaa003.

42. Neuman HB, Elkin EB, Guillem JG, et al. Treatment for patients with rectal cancer and a clinical complete response to neoadjuvant therapy: a decision analysis. Dis Colon Rectum. 2009;52(5):863-71. https://doi.org/10.1007/DCR.0b013e31819e efba.

43. Rao C, Sun Myint A, Athanasiou T, et al. Avoiding radical surgery in elderly patients with rectal cancer is cost-effective. Dis Colon Rectum. 2017;60(1):30-42. https://doi.org/10.1097/DCR. 0000000000000708.

44. Smith FM, Rao C, Oliva Perez R, et al. Avoiding radical surgery improves early survival in elderly patients with rectal cancer, demonstrating complete clinical response after neoadjuvant therapy: results of a decision-analytic model. Dis Colon Rectum. 2015;58(2):159-71. https://doi.org/10.1097/DCR. 0000000000000281.

45. Kim JC, Yu CS, Lim SB, Kim CW, Kim JH, Kim TW. Abdominoperineal resection and low anterior resection: comparison of long-term oncologic outcome in matched patients with lower rectal cancer. Int J Colorectal Dis. 2013;28(4):493-501. h ttps://doi.org/10.1007/s00384-012-1590-8.

46. Konanz J, Herrle F, Weiss C, Post S, Kienle P. Quality of life of patients after low anterior, intersphincteric, and abdominoperineal resection for rectal cancer-a matched-pair analysis. Int $J$ Colorectal Dis. 2013;28(5):679-88. https://doi.org/10.1007/s003 84-013-1683-Z.

47. Marijnen CA, Kapiteijn E, van de Velde CJ, et al. Acute side effects and complications after short-term preoperative radiotherapy combined with total mesorectal excision in primary rectal cancer: report of a multicenter randomized trial. J Clin Oncol. 2002;20(3):817-25. https://doi.org/10.1200/JCO.2002.20.3.817.

48. Moghadamyeghaneh Z, Phelan M, Smith BR, Stamos MJ. Outcomes of open, laparoscopic, and robotic abdominoperineal resections in patients with rectal cancer. Dis Colon Rectum. 2015;58(12):1123-9. https://doi.org/10.1097/DCR. 0000000000000475 .

49. Jayne D, Pigazzi A, Marshall H, et al. Effect of robotic-assisted vs conventional laparoscopic surgery on risk of conversion to open laparotomy among patients undergoing resection for rectal cancer: the ROLARR randomized clinical trial. JAMA. 2017;318(16):1569-80. https://doi.org/10.1001/jama.2017.7219.

50. Murrell ZA, Dixon MR, Vargas H, Arnell TD, Kumar R, Stamos MJ. Contemporary indications for and early outcomes of abdominoperineal resection. Am Surg. 2005;71(10):837-40.

51. Marco MR, Zhou L, Patil S, et al. Consolidation mFOLFOX6 chemotherapy after chemoradiotherapy improves survival in patients with locally advanced rectal cancer: final results of a multicenter phase II trial. Dis Colon Rectum. 2018;61(10):1146-55. https://doi.org/10.1097/DCR. 0000000000001207.

52. Wali AR, Brandel MG, Santiago-Dieppa DR, et al. Markov modeling for the neurosurgeon: a review of the literature and an introduction to cost-effectiveness research. Neurosurg Focus. 2018;44(5):E20. https://doi.org/10.3171/2018.2.FOCUS17805.

53. Ikoma N, You YN, Bednarski BK, et al. Impact of Recurrence and Salvage Surgery on Survival After Multidisciplinary Treatment of Rectal Cancer. J Clin Oncol. 2017;35(23):2631-8. h ttps://doi.org/10.1200/JCO.2016.72.1464.

54. DMEPOS Fee Schedule. US Centers for Medicare \& Medicaid Services. 2020. Available at: https://www.cms.gov/Medicare/M edicare-Fee-for-Service-Payment/DMEPOSFeeSched/DMEPOSFee-Schedule

55. Duncan I, Ahmed T, Dove H, Maxwell TL. Medicare Cost at End of Life. Am J Hosp Palliat Care. 2019;36(8):705-10. https://doi. org/10.1177/1049909119836204.

Publisher's Note Springer Nature remains neutral with regard to jurisdictional claims in published maps and institutional affiliations. 